\title{
PROSPECTS FOR EDGE CURRENT DENSITY DETERMINATION USING LIBEAM ON DIII-D
}

\author{
by \\ D.M. THOMAS, A.S. BOZEK, T.N. CARLSTROM, D.K. FINKENTHAL, ${ }^{*}$ \\ R. JAYAKUMAR, ${ }^{\dagger}$ M.A. MAKOWSKI, ${ }^{\dagger}$ D.G. NILSON, ${ }^{\dagger}$ T.H. OSBORNE, \\ B.W. RICE, ${ }^{\dagger}$ and R.T. SNIDER
}

This is a preprint of a paper presented at the 13th Topical Conference on High-Temperature Plasma Diagnostics, June 18-22, 2000 in Tucson, Arizona and to be published in Review of Scientific Instruments.

*Palomar Community College, San Marcos, California

tLawrence Livermore National Laboratory, Livermore, California

Work supported by
the U.S. Department of Energy under Contract Nos. DE-AC03-99ER54463
and W-7405-ENG-48

GA PROJECT 30033

AUGUST 2000 


\section{DISCLAIMER}

This report was prepared as an account of work sponsored by an agency of the United States Government. Neither the United States Government nor any agency thereof, nor any of their employees, make any warranty, express or implied, or assumes any legal liability or responsibility for the accuracy, completeness, or usefulness of any information, apparatus, product, or process disclosed, or represents that its use would not infringe privately owned rights. Reference herein to any specific commercial product, process, or service by trade name, trademark, manufacturer, or otherwise does not necessarily constitute or imply its endorsement, recommendation, or favoring by the United States Government or any agency thereof. The views and opinions of authors expressed herein do not necessarily state or reflect those of the United States Government or any agency thereof. 


\section{DISCLAIMER}

Portions of this document may be illegible in electronic image products. Images are produced from the best available original document. 


\section{ABSTRACT}

The specific size and structure of the edge current profile has important effects on the MHD stability and ultimate performance of many advanced tokamak (AT) operating modes. This is true for both bootstrap and externally driven currents that may be used to tailor the edge shear. Absent a direct local measurement of $\mathrm{j}(\mathrm{r})$, the best alternative is a determination of the poloidal field. Measurements of the precision $\left(0.1-0.01^{\circ}\right.$ in magnetic pitch angle and 1-10 ms) necessary to address issues of stability and control and provide constraints for EFIT are difficult to do in the region of interest $(\rho=0.9-1.1)$. Using Zeeman polarization spectroscopy of the $2 \mathrm{~S}-2 \mathrm{P}$ lithium resonance line emission from the DIII-D LIBEAM, ${ }^{1,2}$ measurements of the various field components may be made to the necessary precision in exactly the region of interest to these studies. Because of the negligible Stark mixing of the relevant atomic levels, this method of determining $j(r)$ is insensitive to the large local electric fields typically found in enhanced confinement ( $\mathrm{H}$-mode) edges, and thus avoids an ambiguity common to Motional Stark Effect (MSE) measurements of B. Key issues for utilizing this technique include good beam quality, an optimum viewing geometry, and a suitable optical pre-filter to isolate the polarized emission line. A prospective diagnostic system for the DIII-D AT program will be described. 



\section{INTRODUCTION}

The desire to optimize the performance of toroidal plasma confinement systems has led to substantial improvement in tokamak operating parameters over the past few years. The ability to create and maintain transport barriers at various positions in the plasma has led to record levels of confinement. At present, the performance of these "advanced tokamak" modes of operation is set more by the stability limits of various MHD modes (peeling, ballooning, and kink) than by transport considerations. 3,4 Avoidance and extension of these limits require careful tailoring of the plasma pressure and current profiles. As a prime example, the confinement and performance of ELMing $\mathrm{H}$-mode discharges depends critically on the edge pedestal height. Given the proper plasma shape and edge current density, it is possible in the pedestal region to access the second regime of plasma stability for high $\mathrm{n}$ modes, in particular ideal MHD ballooning modes. This allows the edge pressure gradient to increase to values well in excess of the first regime stability limits. ${ }^{5}$ The pedestal height is ultimately limited by low to medium $\mathbf{n}(<10)$ peeling modes. The stability of these relatively long wavelength modes is dependent on the details of the pressure gradient and current density profiles. Hence, detailed measurements of the edge current density are necessary to further develop the physics of the pedestal region and improve the plasma performance of these devices.

Measurement of the current density profile through the use of the Motional Stark Effect (MSE) ${ }^{6}$ has played a key role in the understanding and development of these advanced operational modes. In MSE, the poloidal magnetic field $\mathrm{B}_{\theta}$ (and thus the current density) is typically inferred from the measured polarization of the Doppler-shifted $D_{\alpha}$ emission from an injected hydrogenic neutral beam. Both heating beams and dedicated diagnostic neutral beams have been employed for this purpose. The polarization of the Stark-split emission manifold is determined by the electric field in the rest frame of the beam. For tangential views and radial injection, this has historically been identified with the cross product of beam velocity and total magnetic field: 


$$
E=v_{B E A M} \times B
$$

A complication occurs for most advanced tokamak modes, where substantial intrinsic radial electric fields are found to be coexistent with the high gradients that are often formed by the transport barriers. In this case Eq. (1) generalizes to

$$
\mathrm{E}_{\mathrm{TOT}}=\mathrm{v}_{\mathrm{BEAM}} \times \mathrm{B}+\mathrm{E}_{\mathrm{r}}
$$

The implications of this effect have been discussed in detail in several papers. ${ }^{7-9}$ One specific consequence is the inability of a simple MSE-style measurement to distinguish between the two electric terms in Eq. (2) without substantial modification of the diagnostic (multiple views, multiple beam injection angles, multiple beam energy components, etc). This is of particular import for studies of the edge bootstrap current on DIII-D where small poloidal magnetic field changes $(\sim 1 \%)$ need to be resolved in the presence of large $(\sim 100 \mathrm{kV} / \mathrm{m})$ electric fields associated with high-performance $\mathrm{H}$-modes.

As an improved measurement for this edge region, we are designing a diagnostic based on the Zeeman effect in the lithium $2 \mathrm{~S}-2 \mathrm{P}$ resonance transition. In this case, because of the wide separation of the atomic levels there is no Stark mixing and the polarization and splitting of the resonance emission is strictly due to the local magnetic field. ${ }^{10}$ In this paper we describe some of the requirements for such a measurement on DIII-D and our present approach to deployment. 


\section{PRINCIPLE OF MEASUREMENT}

Diagnostic systems based on lithium Zeeman spectroscopy/polarimetry have been employed on numerous magnetic confinement experiments through the years, ${ }^{11-14}$ using a variety of beam injection and polarization analysis techniques. Using a beam permits localized measurements by using a transverse viewing geometry and well defined viewing volumes.

Once inside the magnetized plasma, the neutral lithium $(2 \mathrm{~S}-2 \mathrm{P}, 1=670.8 \mathrm{~nm}$ ) resonance emission is split with the line separation given by the Zeeman effect $(0.021 \mathrm{~nm}$ per Tesla in the case of lithium for fields greater than about $1 \mathrm{~T}$ ) and broadened due to Doppler effects in the beam. The resulting line profile is a Lorenz triplet, consisting of a central $\pi(\Delta \mathrm{m}=0)$ component and two $\sigma(\Delta \mathrm{m}= \pm 1)$ components, (Fig. 1). The polarization of the three components $(\sigma-, \pi$, and $\sigma+$ ) are determined by the orientation of the local magnetic field and the line of sight. For emission perpendicular to $\mathrm{B}$, the $\pi$ line is linearly polarized parallel to $\mathrm{B}$ while the two $\sigma$ lines are linearly polarized perpendicular to $\mathrm{B}$. For emission parallel to $\mathrm{B}$, there is no $\pi$ line and the two $\sigma$ lines are circularly polarized, with the shorter wavelength $\sigma$ - being left circularly polarized and the longer wavelength $\sigma+$ being right circularly polarized. In terms of Stokes parameters, the general emission state for the line profile ( $\pi$ plus $\sigma+$ plus $\sigma-$ ) can be expressed as a function of the field inclination angle $\gamma$ and the viewing angle $\alpha(\gamma)$ relative to the field

$$
\begin{aligned}
& {[\mathrm{I}]=[\pi]+\left[\sigma^{+}\right]+\left[\sigma^{-}\right]}
\end{aligned}
$$

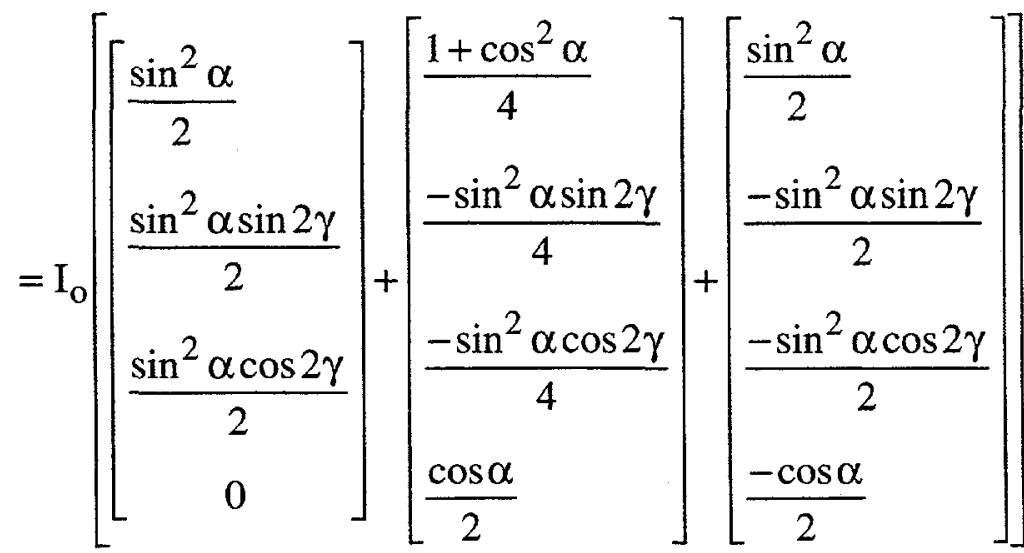




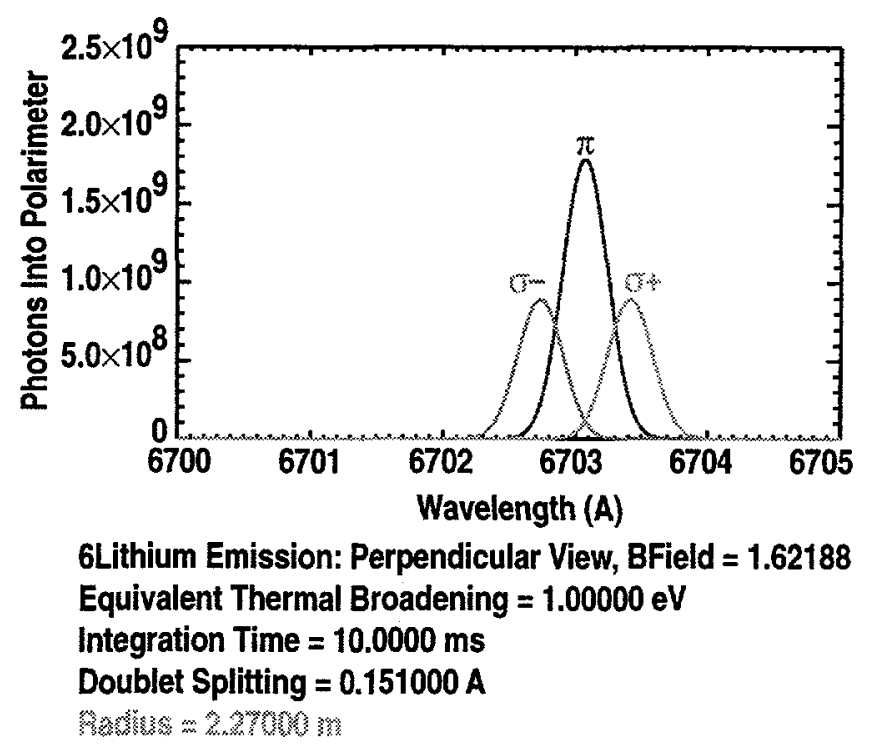

Fig. 1. Lithium line emission profile, calculated for a position near the outside midplane of DIII-D for a $2 T, 1.86$ MA shot. The estimated transverse beam temperature, which dominates the Doppler broadening of the line components, was set at $1.0 \mathrm{eV}$. The distance between the two sigma peaks for this case (total $B=1.62 \mathrm{~T}$ ) is $0.068 \mathrm{~nm}$.

The primary distinction between the lithium Zeeman components and the hydrogenic Stark components is the existence of circular/elliptical polarization for the $\sigma$ states. Polarization analysis of a portion of the line profile (either $\pi$ or $\sigma$ ) can be done using either dynamic [using a photoelastic modulator (PEM)] or static [using discrete polarizing elements and amplitude splitting] techniques, as pointed out by Voslamber. ${ }^{15}$ These measurements (basically intensity ratios between various Stokes components) then allow one to interpret pitch angles, and thence field ratios, poloidal field profiles, and current density. The four keys to a useful measurement are: sufficient intensity to permit the desired spatial and time resolution; accurate spatial calibration of the viewing geometry to permit precise angular determination of the polarization, minimization of unwanted systematic polarization effects, and sufficient spectral rejection of part of the line profile to ensure a reasonable level of polarization in the observed ( $\pi$ or $\sigma$ ) component. 


\section{SENSITIVITY STUDY}

Modeling studies on DIII-D edge plasma indicate the bootstrap current occupies a very small spatial region in the outer part of the plasma. ${ }^{16}$ Hence, a premium is set on radial resolution in the edge. Our previous experience with LIBEAM $^{1}$ allows us to model accurately the beam penetration and brightness on DIII-D for a variety of plasma parameters, viewing geometry, and collection efficiency (Fig. 2). For most plasma shapes commonly run on DIII-D, there is a good match between the location of the peak of the emission profile and the region of strong pressure gradient, where the edge bootstrap current is expected to be. Based on these studies, a radial array of 32 channels with a spot size of $5 \mathrm{~mm}$ appears to give a good compromise between adequate spatial resolution and signal level.

Using this hypothetical array, a sensitivity study was done to assess how much the additional pitch angle measurements would constrain the EFIT reconstructions of plasma current distribution. ${ }^{17}$ For this case, we chose spatial error bars for the measurement of about twice the spot size (to account for non perpendicular beam views), a statistical error of $0.1-0.8^{\circ}$ (based on the relative emission intensities along the beam), and systematic error of $0.1^{\circ}$ (comparable to the existing MSE apparatus). The customary error bars (typically $1 \%-2 \%$ and $3 \mathrm{~mm}$ ) for the magnetic probes, flux loops, and MSE arrays were also included. The DIII-D case chosen for study was a low-q, low triangularity plasma which was found to have an edge pressure gradient well above the first stable limit to ideal ballooning modes. 5 The relatively high pedestal density $\left(8 \times 10^{19} \mathrm{~m}^{-3}\right)$ and enhanced beam attenuation also makes this a conservative case for evaluating diagnostic performance. Whether or not this discharge has second stable access depends on the value of the edge current density. ${ }^{18}$ To begin with, a series of reconstructions varying the inputs within the presumed error bars for all existing measurements yielded a distribution of (equally valid) current profiles. A ballooning mode stability analysis ${ }^{19}$ was done for two equilibria which most closely matched the upper and lower standard deviation of the current profile distribution. For the existing diagnostic set it was not possible to determine whether the edge could achieve 


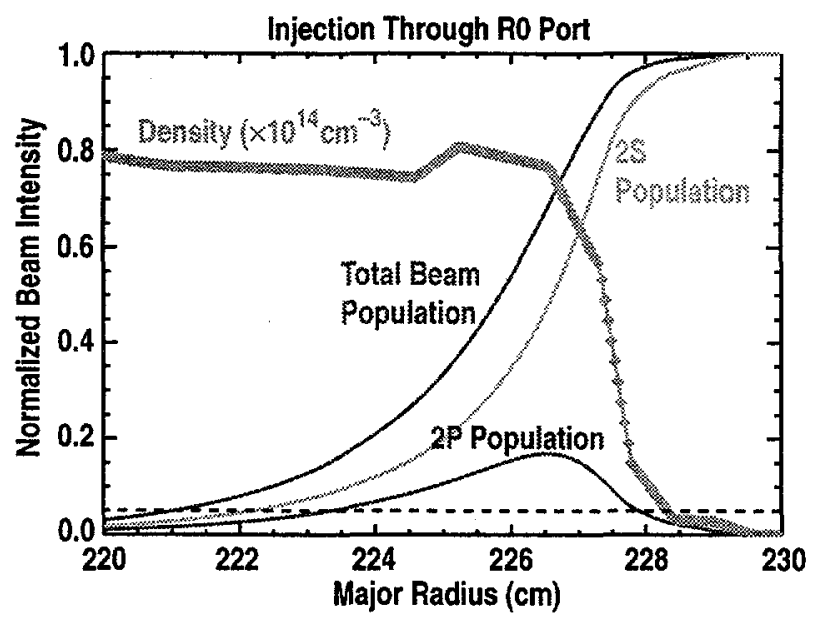

Fig. 2. Results of LIBEAM beam penetration/emission modeling for DIII-D shot \# 98264, during late H-mode prior to a giant ELM. Pedestal density is $8 \times 10^{20} \mathrm{~m}^{-3}$. Shown are the relative total, ground state $2 S$, and excited state $2 P$ populations as a function of major radius. Radial region shown corresponds to a poloidal flux range $0.7<\psi<1.05$. The $5 \%$ excited state level (dashed line) corresponds to a photon rate of $\sim 5 \times 10^{10} \mathrm{~s}^{-1}$ for the beam and viewing parameters cited in the paper.

second stable access or not, due to the uncertainty in edge current. The reconstructions were then repeated, this time including the hypothetical lithium beam array. In this case, the lithium beam measurements significantly improved the reconstruction performance, much more closely constrained the edge current distribution, and clearly allowed us to distinguish second-stable cases from first stable ones (Fig. 3).
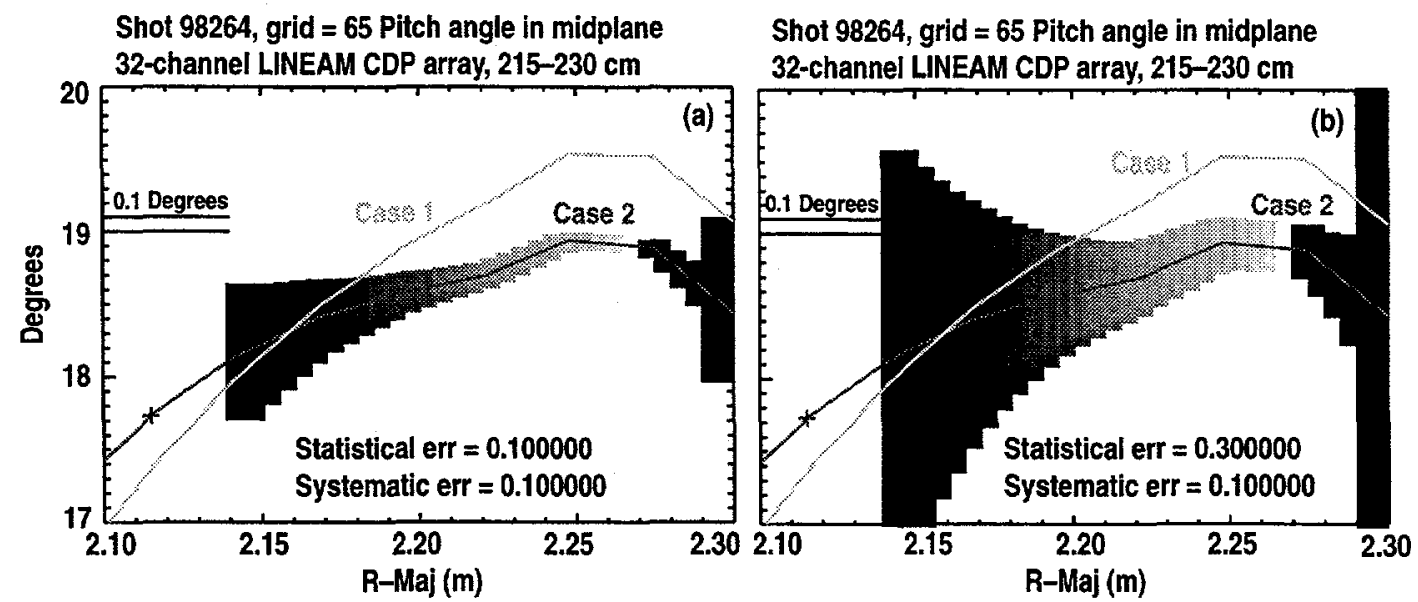

Fig. 3. (a) Error bar matrix of a hypothetical 32-channel lithium beam measurement for shot 98264, presuming a systematic pitch angle measurement error of $0.1^{\circ}$ in quadrature with a relative statistical error of $0.1^{\circ}$, scaled to the $10 \%$ excited state light level. Radial error is set by view spot size, beam height, and off-vertical observation angles. Also shown are pitch angle profiles extracted from EFIT solutions for two different cases cited in the text. Case 2 is second stable according to BALLOO code and case 1 is not, and are indistinguishable using the existing diagnostic measurements. (b) Error bar matrix presuming a systematic error of $0.1^{\circ}$ and a relative statistical error of $0.30^{\circ}$. 


\section{DIAGNOSTIC LAYOUT AND CHOICE OF GEOMETRY}

The planned layout for the diagnostic, at the $75^{\circ}$ toroidal location on DIII-D is shown in Fig. 4. The radial/horizontal injection geometry chosen is similiar to that of the original LIBEAM installation. ${ }^{1}$ LIBEAM operational parameters are: beam energy $30 \mathrm{keV}$, neutral equivalent current $10-20 \mathrm{~mA} \mathrm{CW}$. The use of large area thermoemissive ion sources and sodium vapor neutralization yields a very stable beam with low intrinsic fluctuation and thermal spread. ${ }^{20}$ The expected beam dimension of $1-2 \mathrm{~cm}$ permits the desired spatial resolution using either tangential or vertical views. Alternate injection geometries (for example, vertical, near the separatrix) were considered but ultimately abandoned as being too difficult, providing only modest additional information). Port access dictated the use of a somewhat lower beam trajectory $\left(\mathrm{z}_{\text {beam }}=-12.7 \mathrm{~cm}\right)$ than used previously. Because of the desire for minimal vessel modification, a vertical view was selected as it yields better spatial resolution for a given emission volume than was achievable with the existing tangential viewport. Because of the emission anisotropy for the various states, this view also gives greater sensitivity to the poloidal field. The two drawbacks to this geometry are the need for internal, polarization-maintaining optics, and the requirement of analysing linear and circular polarization components simultaneously. This last requirement is easy to accomplish using conventional PEM technology by making lockin measurements at both the fundamental and second harmonic frequencies. ${ }^{21}$ 


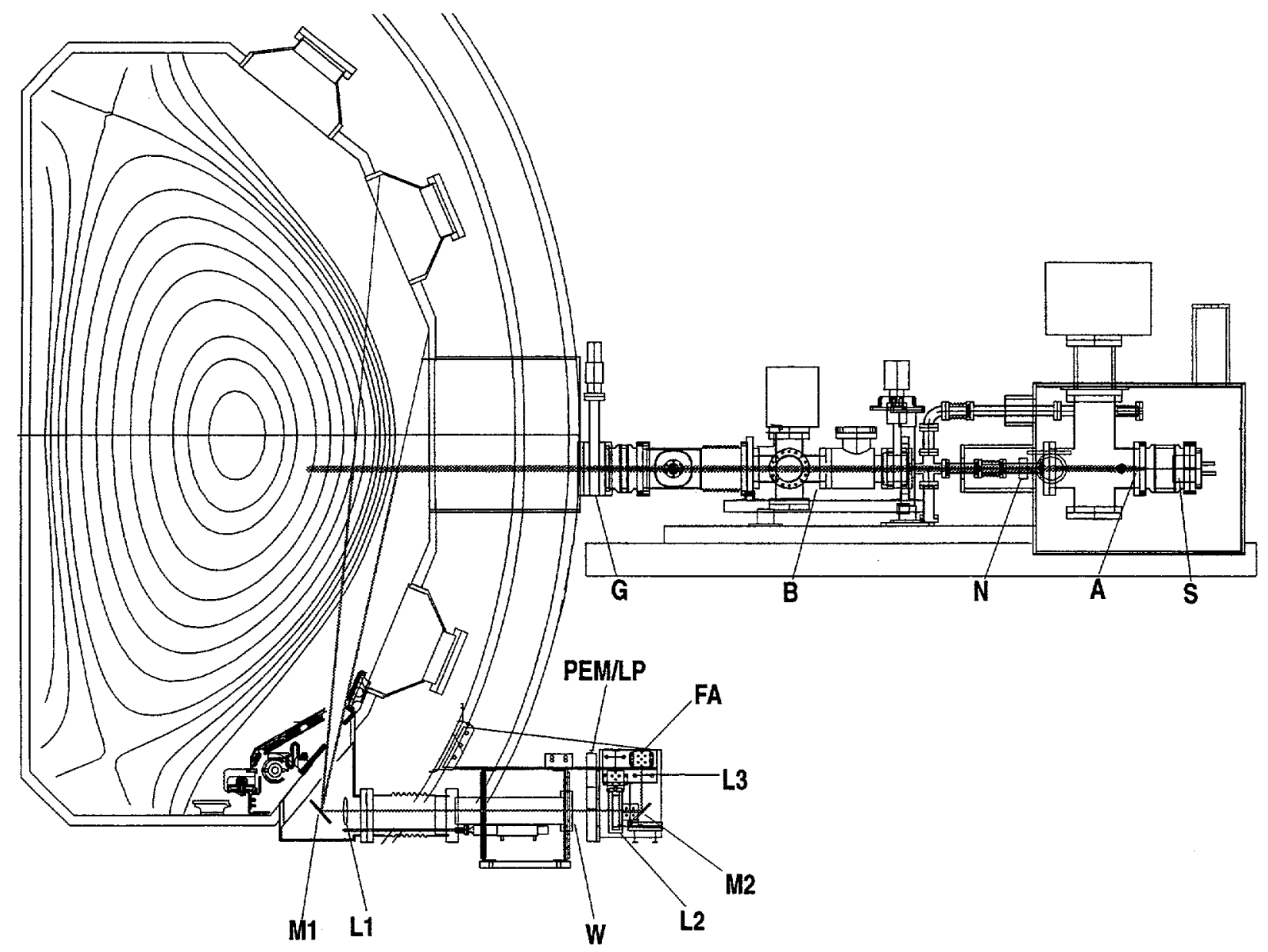

Fig. 4. Cross section of DIII-D showing the planned layout for the accelerator and polarimeter installation. $S$-Source, A-Accelerator, N-Neutralizer, B-Beamline, G-Gate Valve, M1-Dielectric in-vessel Mirror, LI-in-vessel relay Lens, W-Window, PEM-photoelastic modulator pair, LP, linear polarizer, L2-field lens, M2-bending mirror, L3-cylindrical mirror, FA-fiber optic array. 


\section{OPTICAL SYSTEM}

A layout of the proposed optical system is also shown in Fig. 4. Again, the system design was driven by the use of an existing port ("R-2" port) directly beneath the beam trajectory. Because of the shape of the port, internal optical components are required, but can be recessed beneath the floor baffle plate to reduce heating and degradation effects. The 32 viewchords lie in a vertical plane and image the beam at near vertical angles (inclination angle from beam $\alpha=$ $97.3^{\circ}-103.2^{\circ}$ ) The mirror M1 is a multilayer dielectric mirror having minimal phase delay between reflected s- and p- states. The relay lens L1 is used to decrease the effective $f$-number of the system to around $f / 17$. It also allows us to utilize the central section of the PEM aperture, avoiding the phase distortion that occurs near the edges of these devices. ${ }^{22}$ In order to avoid systematic polarization errors due to uncompensated magnetooptic effects (Faraday rotation), this lens must be fabricated of a special, low Verdet constant glass. ${ }^{23}$ Similar care must be taken with the vacuum window W. In this case, the brittleness of the SF-6 glass complicates its use for large diameter windows. We are looking into the feasibility of using a focussing mirror for M1. If we can obtain the proper dielectric coating on a curved mirror this would allow us to eliminate the in vessel relay lens.

The pair of PEMs and analyzer LP1 are located immediately outside of the vacuum window, followed by a field lens L2 and bending mirror M2, used to avoid obstructions just outside of the R-2 port region. A final cylindrical lens L3 is used to image the beam onto an array of $1 \mathrm{~mm}$ diameter fibers FA which carry the light out of the machine pit to the detection room. The use of the cylindrical lens L3 (axis parallel to fiber array) will allow for collection of more emission across the lateral (toroidal) width of the beam, while maintaining the $5 \mathrm{~mm}$ radial spotsize. The useful beam width will ultimately be set by the effective f/number of the fiber for long distances. We expect to be able to achieve a factor of 4 in cylindrical demagnification, permitting light collection from a $2 \mathrm{~cm}$ wide by $5 \mathrm{~mm}$ long beam slice. 
We cannot use off the shelf narrowband optical filters for isolation of the various line components, because of the small Zeeman splitting. While Fabry-Perots are an acceptable, if low transmission solution, we are investigating the use of Lyot filters. 24,25 These birefringent optical elements are capable of much higher effective transmission and acceptance angle compared to Fabry-Perot etalons while offering exquisite spectral resolution. 26

At present we are still deciding between PMTs and off the shelf photodiode detectors as the detection element. Because of previous experience with radiation induced noise on the detection systems of other DIII-D optical diagnostics 27 we are taking some pains to locate the detectors some distance away from the DIII-D shield wall, as well as providing space for future lead/polyborate shields, should they prove to be necessary.

Because of the number of polarization-sensitive elements inside the vessel it will be important to perform in-situ measurements of polarization effects on the optical system to account for systematic effects as completely as possible. We plan to implement retractable polarization elements as part of the shutter assembly for general testing, as well as performing detailed calibrations during vents. Measurements on beam-into-gas fluorescence with toroidal field only should allow us to correct for most of the systematic polarization shifts. Our standard procedure will be to acquire data during toroidal field flattop before discharge initiation, utilizing the gas prefill to excite the beam. The lower signal level can be compensated for by averaging for hundreds of ms, if necessary. In this way, an accurate pitch angle offset should be archived for each channel, every shot. This correction is facilitated by our ability to run the lithium beam independent of plasma operations.

In summary, we are pursuing the reinstallation of the LIBEAM diagnostic beam, with a suitable multichannel polarimeter system, in order to improve our determination of the edge current density on DIII-D. Such measurements are crucial for improved understanding and eventual control of AT modes of operation. The installation of the accelerator, optical system, and machine interfaces on DIII-D is presently planned for completion during the machine vent in fall 2000. The geometry chosen for the diagnostic is also fully compatible with installation of a 
collinear (counterpropagating) pump laser in a future upgrade. This capability would allow us to take advantage of laser induced fluorescence to enhance the collisionally generated emission and should enable magnetic field measurements on open field lines where negligible collisional signal is available .28 



\section{REFERENCES}

${ }^{1}$ D.M. Thomas, Rev.Sci.Instrum. 66, 1, 806 (1995).

2D.M. Thomas, A.W. Hyatt, and M.P. Thomas, Rev. Sci Instrum. 61, 10, 3040 (1990).

${ }^{3}$ L.L. Lao, J.R. Ferron, R.L. Miller, T.H. Osborne, V.S. Chan, R.J. Groebner, G.L. Jackson, R.J.

La Haye, E.J. Strait, T.S. Taylor, A.D. Turnbull, E.J. Doyle, E.A. Lazarus, M. Murakami, G.R.

McKee, B.W. Rice, C. Zhang, L. Chen, Nuc. Fusion 39, 11Y, 1785 (1999).

${ }^{4}$ C.M. Greenfield, et.al., Phys. Plasmas 7, 5, 1959, (2000).

5T.H. Osborne, R.J. Groebner, L.L. Lao, A.W. Leonard, R. Maingi, R.L. Miller, G.D. Porter,

D.M. Thomas, and R.E. Waltz, Plasma Phys. Control. Fusion 40, 845 (1998).

6F. Levinton et al., Phys. Rev. Lett. 63, 2060 (1989).

${ }^{7}$ B.W. Rice, K.H. Burrell, L.L Lao, Nuc. Fusion. 37, 4, 517 (1997).

${ }^{8}$ B.W. Rice, K.H. Burrell, L.L Lao, Y.R. Lin-Liu, Phys. Rev. Lett. 79, 14, 2694 (1997).

${ }^{9}$ M.C. Zarnstorff, F.M. Levinton, S.H. Batha, and E.J. Synakowski, Phys. Plasma 4, 1097 (1997).

${ }^{10}$ H.A. Bethe and E.E. Salpeter, Quantum Mechanics of One- and Two-Electron Atoms. (Plenum, 1977) $205 \mathrm{ff}$.

11K. McCormick and J. Olivain, Rev. Phys. Appl. 13, 85 (1978).

${ }^{12}$ K. Kadota, C., Takehashi, H. Iguchi, M. Fujiwara, K. Matsunaga, and J. Fujita, Rev. Sci. Instrun. 56, 5, 857 (1985).

${ }^{13}$ K. McCormick, F.X. Soldner, D. Eckhartt, F. Leuterer, H. Murmanm, H. Derfler, A. Eberhagen, O. Gehre, J. Gernhardt, G.v. Gierke, O. Gruber, M. Keilhacker, O. Kluber, K. Lackner, D. Meisel, V. Mertens, H. Rohr, K.-H. Schmitter, K.-H. Steuer, and F. Wagner, Phys. Rev. Lett. 58, 5, 491 (1987).

${ }^{14}$ W.P.West, D.M. Thomas, J.S. DeGrassie, and S.B. Zheng, Phys. Rev. Lett. 58, 26, 2758 (1987).

15D. Voslamber, Rev. Sci. Instrum. 66, 2892, (1995).

${ }^{16} \mathrm{M}$. Murakami, et al., Nucl. Fusion, in press. 
${ }^{17} \mathrm{~T}$. Osborne, private communication or DIII-D Physics memo D3DPM-0004(2000).

18J.R. Ferron, M.S. Chu, G.L. Jackson, L.L. Lao, R.L. Miller, T.H. Osborne, P.B. Snyder, E.J. Strait, T.S. Taylor, A.D. Turnbull, A.M. Garofalo, M.A. Makowski, B.W. Rice, M.S. Chance, L.R. Baylor, M. Murakami, M.R. Wade, Phys. Plasmas 7, 5, 1976 (2000).

${ }^{19}$ R.L. Miller, Y.R. Lin-Liu, A.D. Turnbull, V.S. Chan, L.D. Pearlstein, O. Sauter, L. Villard, Phys. Plasmas 4, 1062 (1997).

${ }^{20}$ D.M. Thomas and W.P West, Rev. Sci.Instrun. 59, 8, 1735 (1988).

${ }^{21}$ Kemp, J.C., Polarized Light and its Interaction with Modulating Devices - A Methodology Review, Hinds International, Inc.,1987.

22PEM-90 Data Bulletin, Hinds Instruments, Inc, 1993.

${ }^{23}$ Schott FS-6, Schott glass catalog.

${ }^{24}$ B. Lyot, Ann. Astron. 7, 31 (1944).

${ }^{25}$ A.M. Title, Solar Phys. 33, 521(1973); Appl. Opt. 14, 229 (1975); Appl. Opt. 14, 445 (1975); Appl. Opt. 15, 11, 2879 (1975).

26J. Staromlynska, S.M. Rees, M.P. Gillyon, Appl. Opt. 37, 6, 1081 (1998).

${ }^{27} \mathrm{~K}$. Burrell, private communication (1998).

28D.M. Thomas, et al., Bull. Am Phys. Soc. 44, 7, 174 (1999). 


\section{ACKNOWLEDGEMENTS}

We would like to thank J. Kulchar for his efforts on resurrecting the LIBEAM accelerator system and hardware. We are indebted to F. Levinton for first bringing Lyot filters to our attention. The continuing support and encouragement of T.S. Taylor, T.C. Simonen, and R.D. Stambaugh is also gratefully acknowledged. Work supported by U.S. Department of Energy under Contract DE-AC03-99ER54463 and W-7405-ENG-48. 